\title{
Some features of polarization modulation in spectroscopy of optical and photoelectric effects
}

\author{
S.V. Kondratenko*, I.E. Matyash, B.K. Serdega \\ V. Lashkaryov Institute of Semiconductor Physics, National Academy of Sciences of Ukraine \\ 45, prospect Nauky, 03028 Kyiv, Ukraine \\ *Kyiv Taras Shevchenko National University, 64, Volodymyrska str., 01033 Kyiv, Ukraine \\ E-mail: serdega@isp.kiev.ua
}

\begin{abstract}
We analyze usage of modulation of electromagnetic radiation polarization in investigations of optical and photoelectric effects in anisotropic crystals. It is shown that when transmission spectra are studied with the polarization modulation technique, then the results obtained can be represented as a product of two factors. One of them is the derivative of the transmission coefficient with respect to the absorption coefficient, while another characterizes the absorption coefficient anisotropy. Our analysis demonstrates improved information ability of this technique that enables one to explain some previously obtained results.
\end{abstract}

Keywords: polarization modulation, absorption coefficient, transmission coefficient.

Paper received 12.02.04; accepted for publication 17.06.04.

\section{Introduction}

Modulation of electromagnetic radiation polarization used in experimental physics is one of the techniques of differential spectroscopy. These techniques are based on modulation of different physical quantities (magnetic and electric field strengths, strain, temperature, radiation wavelength, etc.). By now they are studied in detail and generalized (see, e.g., [1-3]). However, such radiation parameter as polarization differs essentially from other characteristics of radiation. The distinction is, first of all, that polarization, being a spatial characteristic of electromagnetic wave, is described by a set of quantities, namely, the components of Maxwell Jones or Stokes vectors. Thus, contrary to modulation of other physical quantities (each of which is described by only one parameter), the polarization modulation (PM) has a two-dimensional effect on a wave. This feature (being inessential prima facie) considerably complicates both the experimental PM technique and interpretation of results obtained with it. This seems to be the reason for the fact that no attention has been given to PM, while other differential techniques enjoyed wide popularity. However, there is good reason to believe that application of PM could increase information ability of various measuring instruments. For instance, the PM technique demonstrated high sensitivity to birefringence when studying the photoelasticity effect (even in the case of opaque substances) [4]. One could expect that this could be true also in other cases because the process of PM covers a set of physical parameters. So the objective of our work was to study the PM features bearing in mind the above considerations.

\section{Experiment}

Let us qualitatively consider a process of measurement with the PM technique. We take, as an example, study of linear dichroism in a cubic crystal where anisotropy of dielectric properties is caused by an oriented external action. As is well known, dichroism lies in distinction between absorption coefficients for linearly polarized radiation when the azimuths of the electric component of electromagnetic wave are parallel to different axes of the optical indicatrix of an anisotropic sample. The experimental observation of the effect involves transmission registration for the above two different polarization states. This is made in the spectral range where a difference between the corresponding absorption coefficients is maximal, i.e., the sample is semitransparent. The traditional procedure lied in determination of a difference between the transmission values measured at two polarizer positions, namely, parallel and perpendicular to the optical axis. Due to various physical reasons, sensitivity of such measuring instruments to pleochroism is no less than several percent. 


\section{S.V. Kondratenko et al.: Some features of polarization modulation in spectroscopy of ...}

A considerable (several orders better than the above value) increase of sensitivity is possible in measuring instruments that use modulation of electromagnetic radiation polarization. It is generally considered that such modulation is a periodic action on a wave that leaves its energy constant, while changing its polarization state. This means that modulation frequency is by many orders lower than that of radiation. In this case the polarization state can vary due to phase shift between either two orthogonal components of linearly polarized radiation or two linear components of circularly polarized radiation [2]. When studying linear pleochroism in optical or photoelectric effects, the above two approaches are equivalent to a first approximation. The only important requirement is that, at modulating action on the electromagnetic wave, its polarization be linear in the extreme phases.

Let radiation go through an anisotropic plate in the semi-transparency range, i.e., $\alpha d \approx 1$. Here $\alpha$ is the absorption coefficient and $d$ is the plate thickness in the direction of light propagation. Then an alternating electric signal at modulation frequency appears at the output of a photosensitive instrument used for detection of radiation. This signal is proportional to the difference between the transmission coefficients:

$\Delta T=T\left(\alpha_{\|}\right)-T\left(\alpha_{\perp}\right)$,

that are function of the absorption coefficients $\alpha_{\|}$and $\alpha_{\perp}$ for radiation whose polarization is, respectively, parallel and normal to the optical indicatrix axes.

Using the expression $\Delta \alpha=\alpha_{\|}-\alpha_{\perp}$ for the difference between these two absorption coefficients, one can obtain:

$\Delta T=\frac{T\left(\alpha_{\perp}+\Delta \alpha\right)-T\left(\alpha_{\perp}\right)}{\Delta \alpha} \Delta \alpha$.

It should be noted that in most cases $\Delta \alpha<<\alpha_{\perp}, \alpha_{\|}$. So, using the definition of derivative, one can transform expression (2) into

$\Delta T=\frac{d T}{d \alpha} \Delta \alpha$.

Thus, $\Delta T$ is proportional to the derivative $d T / d$. Taking into account that the absorption coefficient $\alpha$ is function of photon energy $h v$, it is convenient to present the difference $\Delta T$ as a product of two partial derivatives, $\partial T / \partial(h v)$ and $\left(\frac{\partial(h v)}{\partial \alpha}\right)=\left(\frac{\partial \alpha}{\partial(h v)}\right)^{-1}$ :

$\Delta T=\frac{\partial T}{\partial(h v)} \frac{\partial(h v)}{\partial \alpha} \Delta \alpha$.

Let us analyze the physical meaning of Eq.4. First of all, one can see from it the difference between PM and $\lambda$ modulation [1]. It lies in the fact that the result of $\lambda$-modulation appears in expression (4) through the first factor,

$\frac{\partial T}{\partial(h v)}$. It can be obtained by graphical or physical differentiation of the measured spectral dependence $T(h v)$. 196
On the other hand, one can treat the spectral dependence of the difference $T(h v)$ between the transmission coefficients measured with the PM technique as a result of concurrent effect of three factors, namely, the derivative of transmission coefficient with respect to photon energy, $\partial T / \partial(h v)(\lambda$-modulation); the inverse derivative of absorption coefficient with respect to photon energy, $\left(\frac{\partial \alpha}{\partial(h v)}\right)^{-1}$; and parameter of anisotropy, $\Delta \alpha$. Each of the above factors has its own physical meaning. Say, $\Delta \alpha$ is due to anisotropy of dielectric properties caused by external action, and, correspondingly, it contains information on the nature of the studied object response to the external action. This implies that the absorption coefficients features related to the critical points in energy band spectrum (e.g., Van Hove singularities) may manifest themselves in the spectral characteristic of such optical effect as linear dichroism. The transmission coefficient derivative has a methodological sense. It points to the fact that the highest PM signal measured (which is proportional to the dichroism value $\Delta \alpha$ ) is observed in the spectral region where the slope of the transmission coefficient curve is maximal.

\section{Results}

Let us illustrate the above considerations by some experimental results obtained for a silicon sample that was uniaxially strained along the [110] direction. Its spectral dependence of $\Delta T(h v)$ was measured with the PM technique described in [5], while spectral dependence of the transmission coefficient $T(h v)$ was obtained using standard technique (Fig. 1). The dichroism value $\Delta \alpha$ was found using the expression $\Delta \alpha=\left[(1-R)^{2} / d\right] \ln (1+\Delta T / T)$. It was obtained by transformation of expressions that related the transmission coefficient to the reflection coefficient $R$, sample thickness $\mathrm{d}$ and absorption coefficients for two different polarizations. The derivative $\partial T / \partial(h v)$ was then obtained by differentiating the spectral characteristic of the transmission coefficient, $T(h v)$. As a result, one can determine the only unknown factor, $\left(\frac{\partial \alpha}{\partial(h v)}\right)^{-1}$, in the right side of Eq.4. Thus, having measured the characteristics $\Delta T$ and $T(h v)$, one can determine the spectral dependencies $\Delta \alpha(h v)$ and $\frac{\partial \alpha}{\partial(h v)}$.

The determination of dichroism could be also done in another way, if one had a direct technique for physical differentiation of the spectral dependence of absorption coefficient. Having calculated the spectral dependence of absorption coefficient, $\alpha(h v)$, from the well-known expression for transmission characteristic, and then the inverse derivative $\left(\frac{\partial \alpha}{\partial(h v)}\right)^{-1}$, one could obtain (at the same function $\partial T / \partial(h v))$ the spectral dependence of $\Delta \alpha$. The

$S Q O, 7(2), 2004$ 


\section{S.V. Kondratenko et al.: Some features of polarization modulation in spectroscopy of ...}

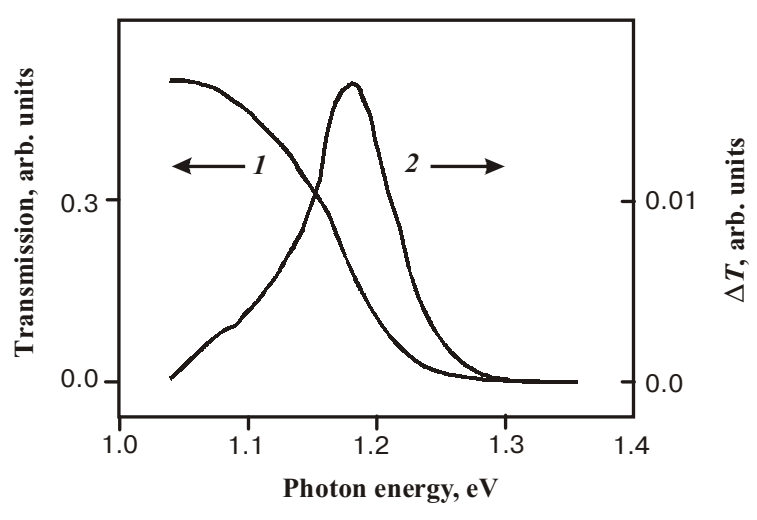

Fig. 1. The transmission coefficient (1) and its variations due to strain (2) as function of photon energy for a Si sample $0.38 \mathrm{~mm}$ thick uniaxially strained along the [110] direction (stress of 0.3 kbar).

latter characterizes variation of the material permittivity with external action.

It seems to be of interest to discuss interrelation between the amplitudes of the factors in the right side of Eq.4. They are shown in Fig. 2 for the same silicon sample [5] uniaxially strained along the [110] direction (stress of $0.3 \mathrm{kbar}$ ). To make the situation clearer, all curves are presented at the comparable scale, in spite of the fact that their ordinate values differ by an order of magnitude. It will be recalled that the product of the ordinate values of these functions gives the curve 2 presented in Fig. 1. This means that in our case the contributions from these three

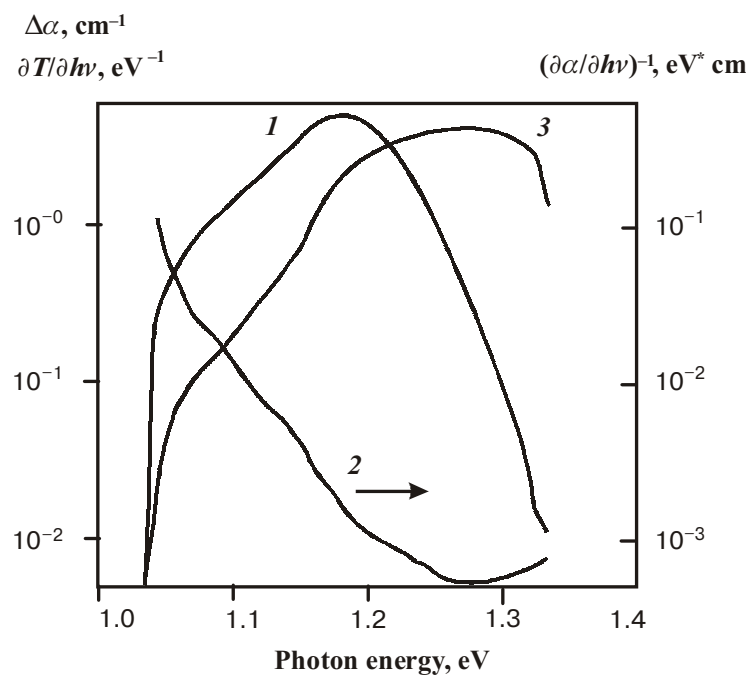

Fig. 2. The spectral dependencies of: the partial derivative of the transmission coefficient, $\partial T / \partial(h v)(1)$; inverse partial derivative of the absorption coefficient, $\left(\frac{\partial \alpha}{\partial(h v)}\right)^{-1}$ (2); difference $\Delta \alpha$ between the absorption coefficients for two orthogonal states of linear polarization (3). (Calculated from the expressions given in the text, with use of the experimental results from Fig.1.)

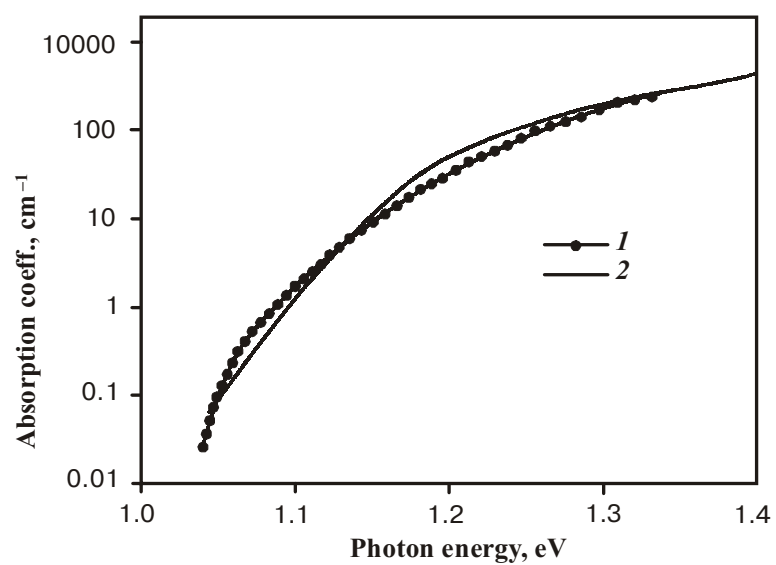

Fig. 3. The absorption coefficient $\alpha$ as function of photon energy $h v$ (calculated by integration of $\partial \alpha / \partial(h v))(1))$ as compared to the results obtained in [6] (2).

factors to $\Delta T$ value (that is measured experimentally) are comparable. The peculiarities and non-monotonic features of the same physical origin coincide for the curves 2 and 3 (Fig. 2) only. In particular, the position of the peak of dichroism curve coincides with that of the minimum of $\partial(h v) / \partial \alpha$ function. As to the curve 1 , it characterizes the sample properties, that is, the transmission coefficient dispersion that depends on the sample thickness. This means that for such crystals like GaAs (where the absorption curve edge is not as lengthy as in silicon and, correspondingly, the slope of the transmission coefficient curve is greater) the results of measurements obtained with the PM technique are determined by the transmission coefficient dispersion to a greater extent, all other factors being equal.

Let us illustrate the adequacy of the advanced method of analysis by comparing the results it gives with independent data. To this end, we integrate the calculated function $\partial(h v) / \partial \alpha$ (curve 2 in Fig. 2) with respect to the photon energy. The obtained spectral characteristic of the absorption coefficient $\alpha$ as a function of photon energy is shown in Fig. 3 (curve 1 ) along with the similar curve from [6]. The agreement between these two curves is more than satisfactory. This fact seems to be decisive evidence that our consideration is adequate.

\section{Conclusions}

The above approach to treatment of spectral characteristics taken with the PM technique was used when considering linear dichroism. It seems obvious that the same approach can be applied to consideration of circular dichroism. For its measurement a modulator based on the photoelastic effect [7] can be used. This instrument can periodically vary direction of circular polarization of electromagnetic wave. Similar argumentation can be extended to usage of this PM technique for measurements of other effects related to radiation absorption in semiconductors, such as photoconductivity, photovoltaic ef- 


\section{S.V. Kondratenko et al.: Some features of polarization modulation in spectroscopy of ...}

fects, etc. For instance, photoconductivity is measured with the PM technique, then the transmission coefficient $T$ in Eq. (3) should be replaced by photocurrent $I_{p c}(\alpha)$.

The results of photopleochroism studies [8] can serve as support of the above statements. In this work, a spectral characteristic of photopleochroism for a crystal with intrinsic natural anisotropy is presented. In the first approximation, this characteristic looks like a derivative of the photoconductivity spectral curve. The latter curve has a peak at big values of the surface recombination velocity, so its derivative must be alternating. And just this tooks place in [8]. Contrary to the $\lambda$-modulation spectra, the characteristic obtained with PM technique has a fine structure, which (according to the above expression (4)) carries information on the anisotropy parameter $\Delta \alpha(h v)$. One can separate these two functions that are contained in the presented spectral dependence, if one invokes the results of additional measurements (say, those performed with $\lambda$-modulation).

Thus, we have shown that the results of any polarization effect measurement performed with PM technique contain (contrary to those obtained with all other modulation types) not one, but two functional dependencies (to say nothing of the dichroism parameter that is an essential characteristic of anisotropic crystals).

\section{References}

1. M. Cardona, Modulation Spectroscopy. Academic Press, New York and London, 1969.

2. K.G. Gusev, A.D. Filatov, A.P. Sokolov, Polarization Modulation. Sovetskoe Radio, Moscow, 1974 (in Russian).

3. V.A. Tyagai, O.V. Snitko, Electroreflectance of Light in Semiconductors. Naukova Dumka, Kiev, 1980 (in Russian).

4. I.I. Boiko, Ye.F. Venger, E.V. Nikitenko, B.K. Serdega // Semiconductor Physics, Quantum Electronics \& Optoelectronics, 2(2), pp. 54-58 (1999).

5. E.F. Venger, I.E. Matyash, B.K. Serdega // Opt. Spectrosc., 94, No 1, pp. 38-42 (2003) (in Russian).

6. W.C. Dash, F. Newman // Phys. Rev., 99, pp. 1151-1155, 1955.

7. S.N. Jasperson, S.E. Sahnatterly // Rev. Sci. Instrum., 40, No 6, pp. 761 (1969).

8. G.A. Medvedkin // Fiz. Tekh. Poluprov, 34, No 6, pp. 533536 (2000) (in Russian). 